\title{
Article \\ Studying the Nonlinear Optical Properties of Fluoride Laser Host Materials in the Ultraviolet Wavelength Region
}

\author{
Duong Van Pham ${ }^{1}$, Diep Van Nguyen ${ }^{1,2}{ }^{2}$, Tu Xuan Nguyen ${ }^{1}$, Kieu Anh Thi Doan ${ }^{1}$, Quan Minh Le ${ }^{3}$, \\ Minh Hong Pham ${ }^{1, *}$ and Marilou Cadatal-Raduban ${ }^{4,5}$ iD \\ 1 Institute of Physics, Vietnam Academy of Science and Technology, Hanoi 100000, Vietnam; \\ pvduong@iop.vast.vn (D.V.P.); nguyenxuantu179@gmail.com (T.X.N.); kieuanhdt99@gmail.com (K.A.T.D.) \\ 2 Graduate University of Science and Technology, Vietnam Academy of Science and Technology, \\ Hanoi 100000, Vietnam \\ 3 Faculty of Teacher Education, HaTinh University, 447, 26/3 Street, Dai Nai Ward, Ha Tinh 480000, Vietnam; \\ quan.le@htu.edu.vn \\ 4 Centre for Theoretical Chemistry and Physics, School of Natural and Computational Sciences, \\ Massey University, Auckland 0632, New Zealand; M.Raduban@massey.ac.nz \\ 5 Institute of Laser Engineering, Osaka University, 2-6 Yamadaoka, Suita 565-0871, Osaka, Japan \\ * Correspondence: diepnvd@gmail.com (D.V.N.); phminh@iop.vast.vn (M.H.P.)
}

check for

updates

Citation: Pham, D.V.; Nguyen, D.V.; Nguyen, T.X.; Doan, K.A.T.; Le, Q.M.; Pham, M.H.; Cadatal-Raduban, M. Studying the Nonlinear Optical Properties of Fluoride Laser Host Materials in the Ultraviolet Wavelength Region. Appl. Sci. 2022, 12, 372. https://doi.org/10.3390/ app12010372

Academic Editor: Emiliano Principi

Received: 29 November 2021

Accepted: 27 December 2021

Published: 31 December 2021

Publisher's Note: MDPI stays neutral with regard to jurisdictional claims in published maps and institutional affiliations.

Copyright: (c) 2021 by the authors Licensee MDPI, Basel, Switzerland. This article is an open access article distributed under the terms and conditions of the Creative Commons Attribution (CC BY) license (https:// creativecommons.org/licenses/by/ $4.0 /)$.

\begin{abstract}
Fluoride host materials doped with trivalent cerium ions have previously been demonstrated as successful laser materials in the ultraviolet wavelength region. However, the nonlinear optical properties of the fluoride hosts in this wavelength region have not been investigated yet, although nonlinearity could result in undesirable effects such as self-focusing and pulse distortion when these fluoride materials are used as gain media in high-power, ultrashort pulse laser oscillator and amplifier systems. In this work, the nonlinear refractive index of lithium calcium aluminum fluoride $\left(\mathrm{LiCaAlF}_{6}\right)$, lithium strontium aluminum fluoride $\left(\mathrm{LiSrAlF}_{6}\right)$, lanthanum fluoride $\left(\mathrm{LaF}_{3}\right)$, and yttrium lithium fluoride $\left(\mathrm{YLiF}_{4}\right)$ fluoride host materials are determined using the Kramers-Krönig relation model in the ultraviolet wavelength region. Self-focusing conditions, particularly at the peak laser emission wavelength of these materials, are further analyzed. Results show that $\mathrm{LiCaAlF}_{6}$ has the smallest nonlinear refractive index and self-focusing, making it an ideal host material under the conditions of ultrashort pulse and ultrahigh-power laser generation.
\end{abstract}

Keywords: ultraviolet; laser; fluoride materials; nonlinear refractive index; self-focusing

\section{Introduction}

Ultrashort, high-peak-power lasers such as femtosecond and attosecond lasers have become indispensable in many applications spanning the fields of physics, chemistry, biology, medicine, and engineering [1,2]. Ultrashort lasers operate fundamentally in the infrared region, but there is much demand in the ultraviolet (UV) wavelength region for numerous applications, including time-resolved spectroscopy [3], micromachining for the fabrication of micro-lens arrays, photonic crystals, optical waveguides, gratings, micro-sized optical sensors [4,5], high-efficiency laser processing of materials [6], and high-resolution ultrafast imaging [7]. Traditionally, high-order harmonic generation using nonlinear crystals has been utilized to convert ultrashort laser pulses from the fundamental infrared to UV wavelengths. The conversion efficiency of such a scheme has reached levels sufficient for the aforementioned applications [8]. However, power scaling is limited, the experimental setup is complex, and conversion efficiency is low. On the other hand, direct generation of pulsed tunable UV laser wavelengths has been achieved using trivalent cerium ion $\left(\mathrm{Ce}^{3+}\right)$-doped fluorine-based host materials. Solid-state tunable lasers have been developed using the following host materials doped with $\mathrm{Ce}^{3+}$ ions: yttrium lithium fluoride $\left(\mathrm{YLiF}_{4}\right.$ or $\left.\mathrm{YLF}\right)$ [9], lanthanum fluoride $\left(\mathrm{LaF}_{3}\right.$ of $\left.\mathrm{LaF}\right)$ [10], lithium lutetium fluoride 
$\left(\mathrm{LiLuF}_{4}\right.$ or LLF) [11], lithium calcium aluminum fluoride ( $\mathrm{LiCaAlF}_{6}$ or LiCAF) [12], and lithium strontium aluminum fluoride ( $\mathrm{LiSrAlF}_{6}$ or LiSAF) [13]. Based on the dipole-allowed interconfigurational $5 \mathrm{~d}-4 \mathrm{f}$ transitions in the $\mathrm{Ce}^{3+}$ activator ions, these new class of solidstate tunable UV lasers are efficient, compact, and are especially attractive for ultrashort pulse generation and amplification in the UV wavelength region.

Several works have used these $\mathrm{Ce}^{3+}$-doped laser materials to develop tunable narrow linewidth UV lasers [14] and high-peak-power UV lasers using various amplifier configurations, including multipass amplification [15], direct amplification of femtosecond pulses [16,17], and chirped pulse amplification [18]. Recently, 100 fs UV pulses were directly generated using a synchronously-pumped mode-locked $\mathrm{Ce}^{3+}$-doped LiCAF laser [19]. Despite the considerable success of these $\mathrm{Ce}^{3+}$-doped fluoride laser materials, their full potential has not yet been fully realized. For example, it has been proposed that terawatt UV pulses can be generated directly using $\mathrm{Ce}^{3+}$-doped LiCAF [20], but that is yet to be realized. An ultrashort pulse with high peak intensity propagating in a medium can experience nonlinear optical effects, for example self-focusing, such that the medium acts as a Kerr lens [21]. Self-focusing arises from the dependence of a material's refractive index on the intensity of the laser beam propagating through that material. It has been leveraged to generate ultrashort pulses through Kerr lens mode-locking [22]. However, self-focusing can also have damaging effects, as the laser fluence at the focus could exceed the damage threshold of the laser material as well as the peripheral optical elements. To avoid the damaging effects of self-focusing, parameters such as the length of the laser material and pump beam radius could be adjusted. However, information about the nonlinear refractive index of fluoride materials in the UV region is lacking. Determination of the nonlinear refractive index in the UV region is important for optimizing the parameters of UV lasers and amplifiers to minimize self-focusing.

While doping with rare-earth activator ions, for example $\mathrm{Ce}^{3+}$ activator ion, dictates the spectrum of laser emission, the performance of the laser and amplifier itself is largely influenced by the optical properties of the host crystal. In this paper, the nonlinear refractive index of fluoride materials used as laser and amplifier host materials in the UV region including LiCAF, LiSAF, LaF, and YLF is explored. Moreover, self-focusing of a highintensity laser beam propagating in the fluoride material is also studied theoretically. Our results could inform experiments involving ultrashort, high-power laser generation and amplification in the UV region, potentially paving the way towards achieving terawatt UV pulses while limiting the destructive effects of self-focusing.

\section{Methodology}

The optical Kerr effect is a nonlinear effect whereby the refractive index of a material changes in response to light propagating in the material. For a particular wavelength, the refractive index is proportional to the local irradiance of the light, being widely defined as [23]:

$$
n(\omega)=n_{0}(\omega)+n_{2}(\omega) I,
$$

where $n_{0}(\omega)$ and $n_{2}(\omega)$ are the linear and nonlinear refractive indices at the frequency $\omega$, respectively, and $I$ is the laser light intensity. It follows that $\Delta n_{2}(\omega)=n_{2}(\omega) I$ is the change in the nonlinear refractive index.

The Kramers-Krönig relation model proposed by Ralph Krönig and Hans Kramers predicts the dispersion and the magnitude of the nonlinear refractive index $\left(n_{2}\right)$ of widebandgap materials. Calculation of $n_{2}$ for solid-state oxide materials using the KramersKrönig relation model has excellent agreement with experimental values [24,25]. Hence, we use the same model to calculate $n_{2}$ for the solid-state fluoride laser host materials LiCAF, LiSAF, LaF, and YLF. According to the Kramers-Krönig dispersion relation, the change in the refractive index is given by

$$
\Delta n(\omega)=\frac{c}{\pi} \int_{0}^{\infty} \frac{\Delta \alpha\left(\omega^{\prime} ; \omega\right)}{\omega^{\prime 2}-\omega^{2}} d \omega^{\prime},
$$


where $\Delta n(\omega)$ is the change in the refractive index due to the presence of pump or excitation light of frequency $\omega$. The change in the index is associated with changes in the absorption coefficient $\Delta \alpha\left(\omega^{\prime} ; \omega\right)$ at the frequency $\omega$ throughout the spectrum $\omega$ ' [24]. The equation for calculating the nonlinear refractive index $n_{2}$ can be derived from the Kramers-Krönig dispersion relation in Equation (2), as discussed in detail by M. Sheik-Bahae et al. [24] and given in Equation (3), as follows:

$$
n_{2}(\omega)=\frac{4 \times 10^{5} \pi K \sqrt{E_{p}}}{c n_{0}^{2}(\omega) E_{g}^{4}} G_{2}\left(\frac{\hbar \omega}{E_{g}}\right)
$$

where $E_{g}$ is the bandgap of the material $(\mathrm{eV}), n_{0}$ is the linear refractive index of the material which can be calculated using the material's Sellmeier equation, $c$ is the speed of light $(\mathrm{cm} / \mathrm{s}), E_{p}$ is a Kane momentum parameter that has a value of $21 \mathrm{eV}, K$ is a materialindependent constant equal to $0.86 \times 10^{-8}$ when $E_{g}$ and $E_{p}$ are in electron volts $(\mathrm{eV})$, and $n_{2}$ is in square centimeters per watt $\left(\mathrm{cm}^{2} / \mathrm{W}\right)$ [24]. The values of these parameters, including the band-gap energies of the laser host materials studied in this paper, are summarized in Table 1.

Table 1. Value of parameters used to solve the nonlinear refractive index $n_{2}$ using Equation (3).

\begin{tabular}{cc}
\hline Kane momentum parameter, $E_{p}$ & $21 \mathrm{eV}[24,25]$ \\
\hline Material-independent constant, $K$ & $0.86 \times 10^{-8}[24,25]$ \\
\hline Speed of light, $c$ & $3.10^{8} \mathrm{~m} / \mathrm{s}$ \\
\hline Planck constant, $\hbar$ & $6.625 \times 10^{-34} \mathrm{J.s}$ \\
\hline Spectral range, $\omega^{\prime}$ & $200-400 \mathrm{~nm}$ \\
\hline Material's band-gap energy (eV) & $12.23[26]$ \\
lithium calcium aluminum fluoride (LiCAF) & $11.79[26]$ \\
lithium strontium aluminum fluoride (LiSAF) & $11.09[27]$ \\
lyttrium lithium fluoride (YLF) & $9.00[28]$ \\
lanthanum fluoride (LaF) &
\end{tabular}

In Equation (3), $G_{2}\left(\hbar \omega / E_{g}\right)$ is a dispersion function given by

$$
G_{2}\left(\frac{\hbar \omega}{E_{g}}\right)=\frac{2}{\pi} \int_{0}^{\infty} \frac{F_{2}\left(x_{1} ; x_{2}\right)}{x_{1}^{2}-x_{2}^{2}} d x
$$

where $x_{1}=\hbar \omega_{1} / E_{g}, x_{2}=\hbar \omega_{2} / E_{g}$, and $F_{2}\left(x_{1} ; x_{2}\right)$ is a nonlinear absorption spectral function that considers the contribution of two-photon absorption (2PA), Raman, and optical Stark effects $[24,29]$. The function $F_{2}\left(x_{1} ; x_{2}\right)$ reflects the band structure of the material and involves only the parameters $x_{1}$ and $x_{2}$; meaning it is a function only of the ratio of the photon energy $\hbar \omega$ to the band gap $E_{g}$ of the material $[25,29]$. Therefore, calculation of the dispersion of the nonlinear refractive index $n_{2}$ requires the band-gap energy of the fluoride host material and the energy of the exciting photon. The expressions of $F_{2}\left(x_{1} ; x_{2}\right)$ for the three contributions are listed in Table 2 [29].

Table 2. Nonlinear absorption spectral function $F_{2}\left(x_{1} ; x_{2}\right)$ for two-photon absorption (2PA), Raman, and optical Stark effects.

\begin{tabular}{cc}
\hline 2PA & $\frac{\left(x_{1}+x_{2}-1\right)^{3 / 2}}{2^{7} x_{1} x_{2}^{2}}\left(\frac{1}{x_{1}}+\frac{1}{x_{2}}\right)^{2}$ \\
$x_{1}+x_{2}>1$ & $\frac{\left(x_{1}-x_{2}-1\right)^{3 / 2}}{2^{7} x_{1} x_{2}^{2}}\left(\frac{1}{x_{1}}-\frac{1}{x_{2}}\right)^{2}$ \\
\hline Raman & \multicolumn{1}{c}{$\left[x_{2}>1\right.$} \\
$x_{1}-x_{1}$ & $\left.\frac{2\left(x_{1}-1\right)\left(x_{1}^{2}+x_{2}^{2}\right)}{\left(x_{1}^{2}-x_{2}^{2}\right)^{2}}+\frac{8\left(x_{1}-1\right)^{2}}{x_{2}^{2}}\right]$ \\
\hline Stark & $-\frac{1}{x_{1}>1}$ \\
\hline
\end{tabular}


Using the function $F_{2}\left(x_{1} ; x_{2}\right)$ in Table 2, the integration in Equation (4) can be performed analytically to obtain the nondegenerate $G_{2}\left(x_{1} ; x_{2}\right)$ and hence also to calculate the nonlinear refractive index $n_{2}$ using Equation (3). Table 3 summarizes the nondegenerate nonlinear dispersion functions for the three contributions [29]. For laser generation, only the degenerate dispersion is useful. Therefore, $n_{2}$ was calculated using $x_{1}=x_{2}=\hbar \omega / E_{g}$. Previous works have reported that this Kramers-Krönig transformation-based model gives good agreement with experimental nonlinear refractive index data for different materials, including oxides such as sapphire [25] and fluorides such as lithium fluoride [24].

Table 3. Nondegenerate dispersion function $G_{2}$ for two-photon absorption (2PA), Raman, and optical Stark effects calculated by a Kramers-Krönig transformation of $F_{2}$.

\begin{tabular}{|c|c|}
\hline $2 \mathrm{PA}$ & $\mathrm{H}\left(x_{1} ; x_{2}\right)+\mathrm{H}\left(-x_{1} ; x_{2}\right)$ \\
\hline Raman & $\begin{array}{c}\mathrm{H}\left(x_{1} ;-x_{2}\right)+\mathrm{H}\left(-x_{1} ;-x_{2}\right) \\
=\frac{1}{2^{6} x_{1}^{4} x_{2}^{4}} \times\left[\begin{array}{c}\text { where for both 2PA and Raman, } \mathrm{H}\left(x_{1}, x_{2}\right) \\
\frac{5}{16} x_{2}^{3} x_{1}^{2}+\frac{9}{8} x_{2}^{2} x_{1}^{2}-\frac{9}{4} x_{2} x_{1}^{2}-\frac{3}{4} x_{2}^{3}-\frac{1}{32} x_{2}^{3} x_{1}^{2}\left(1-x_{1}\right)^{-\frac{3}{2}} \\
+\frac{1}{2}\left(x_{2}+x_{1}\right)^{2}\left[\left(1-x_{2}-x_{1}\right)^{3 / 2}-\left(1-x_{1}\right)^{3 / 2}\right] \\
-\frac{3}{16} x_{2}^{2} x_{1}^{2}\left[\left(1-x_{1}\right)^{-\frac{1}{2}}+\left(1-x_{2}\right)^{-\frac{1}{2}}+\frac{3}{2} x_{2} x_{1}^{2}\left(1-x_{2}\right)^{\frac{1}{2}}\right. \\
+\frac{3}{2} x_{2}^{2} x_{1}\left(1-x_{1}\right)^{\frac{1}{2}}+\frac{3}{4} x_{2}\left(x_{2}^{2}+x_{1}^{2}\right)\left(1-x_{1}\right)^{\frac{1}{2}} \\
-\frac{3}{8} x_{2}^{3} x_{1}\left(1-x_{1}\right)^{-\frac{1}{2}}+\frac{1}{2}\left(x_{2}^{2}+x_{1}^{2}\right)\left[1-\left(1-x_{2}\right)^{\frac{3}{2}}\right]\end{array}\right.\end{array}$ \\
\hline $\begin{array}{l}\text { Stark } \\
x_{1} \neq x_{2}\end{array}$ & $\left.\begin{array}{c}\frac{1}{2^{9} x_{1}^{2} x_{2}^{2}}\left[\begin{array}{c}-\frac{1}{2}-\frac{4}{x_{1}^{2}}+\frac{4}{x_{2}^{2}}-\frac{x_{2}^{2}}{x_{1}} \frac{\left.\left(1-x_{1}\right)^{-\frac{1}{2}}-\left(1+x_{1}\right)^{-\frac{1}{2}}\right]}{x_{1}^{2}-x_{2}^{2}} \\
+\frac{2 x_{1}^{2}\left(3 x_{2}^{3}-x_{1}^{2}\right)}{x_{2}^{2}\left(x_{1}^{2}-x_{2}^{2}\right)^{2}}\left[\left(1-x_{2}\right)^{\frac{1}{2}}+\left(1+x_{2}\right)^{\frac{1}{2}}\right] \\
-\frac{2 x_{2}^{2}\left(3 x_{1}^{3}-x_{2}^{2}\right)}{x_{1}^{2}\left(x_{1}^{2}-x_{2}^{2}\right)^{2}}\left[\left(1-x_{1}\right)^{\frac{1}{2}}+\left(1+x_{1}\right)^{\frac{1}{2}}\right]\end{array}\right] \\
\frac{1}{2^{9} x_{1}^{4}}\left[\frac{3}{4} \frac{\left(1-x_{1}\right)^{-\frac{1}{2}}-\left(1+x_{1}\right)^{-\frac{1}{2}}}{x_{1}}-\frac{\left(1-x_{1}\right)^{-\frac{3}{2}}+\left(1+x_{1}\right)^{-\frac{3}{2}}}{8}-\frac{1}{2}\right.\end{array}\right]$ \\
\hline
\end{tabular}

As mentioned previously, the nonlinear refractive index gives rise to nonlinear optical processes such as self-focusing. This occurs if the energy of the laser pulse in the material is greater than the critical energy, which is defined by [30]

$$
E_{c r}(\lambda)=\alpha \frac{\lambda^{2} \tau}{4 \pi n_{0}(\lambda) n_{2}(\lambda)}
$$

where $\lambda$ is the wavelength of the laser (nm), $\alpha$ is a constant whose value is equal to 1.8962 for a Gaussian beam, $\tau$ is the duration of the laser pulse (s), and $n_{0}$ is the linear refractive index of materials.

Self-focusing could damage the laser material as well as peripheral optical components if the focal point occurs inside the laser material or optical component. When self-focusing occurs, the material acts as a Kerr lens with a defined focal length $f$. Therefore, it is necessary to determine the focal length of the Kerr lens and ensure that the focal point is outside the material to avoid the damaging effects of self-focusing. The inverse focal length $f^{-1}$ is given by [31]

$$
f^{-1}=\frac{8 n_{2}(\lambda) d}{\pi r^{4}} P
$$

where $P$ is the peak power of the laser $(\mathrm{W}), r$ is radius of the laser beam at the surface of the laser material $(\mathrm{mm})$, and $d$ is the length of the laser material $(\mathrm{mm})$.

All calculations were performed using MATLAB software. Calculations were performed in the UV region spanning the wavelength range from $200 \mathrm{~nm}$ to $400 \mathrm{~nm}$, with a step size of $1 \mathrm{~nm}$. 


\section{Results and Discussion}

\subsection{Linear and Nonlinear Refractive Indices of the Fluoride Laser Host Materials}

The linear dispersion of the fluoride materials in the UV region were calculated using their individual Sellmeier equations [32-35] and are shown in Figure 1. As expected, the linear refractive index of the materials decreased for longer wavelengths. Among the four fluoride materials, LiSAF exhibited the smallest variation in its $n_{0}$, changing from 1.44 at $200 \mathrm{~nm}$ to 1.41 at $400 \mathrm{~nm}$, corresponding to a $\Delta n_{0}$ of 0.03 . LiCAF also exhibited a small variation in its $n_{0}$ which changed from 1.43 at $200 \mathrm{~nm}$ to 1.39 at $400 \mathrm{~nm}$ and a $\Delta n_{0}$ of 0.04 . The value of $n_{0}$ at the $290 \mathrm{~nm}$ peak laser emission wavelength is $\sim 1.4$, which is in good agreement with the previously reported experimental values [32]. The refractive index of the doped and undoped materials are similar, differing by only $<1 \%$ of each other. For example, the refractive index of undoped and $\mathrm{Ce}^{3+}$-doped LiCAF at the $290 \mathrm{~nm}$ UV laser emission wavelength is 1.40 [32] and 1.41 [36], respectively. Similarly, the difference in the refractive index between a chromium-doped $\mathrm{LiCAF}\left(\mathrm{Cr}^{3+}: \mathrm{LiCAF}\right)$ and undoped LiCAF is about $5 \times 10^{-3}$ throughout the spectral range from 200-400 nm [37]. YLF had a $\Delta n_{0}$ of 0.05 , with its $n_{0}$ changing from 1.52 to 1.47 at the same wavelength range as the others. Notably, LaF had the largest $\Delta n_{0}$ of 0.08 as its $n_{0}$ ranged from 1.70 at $200 \mathrm{~nm}$ to 1.62 at $400 \mathrm{~nm}$. In general, LaF also had higher $n_{0}$ values compared to the other fluoride materials.

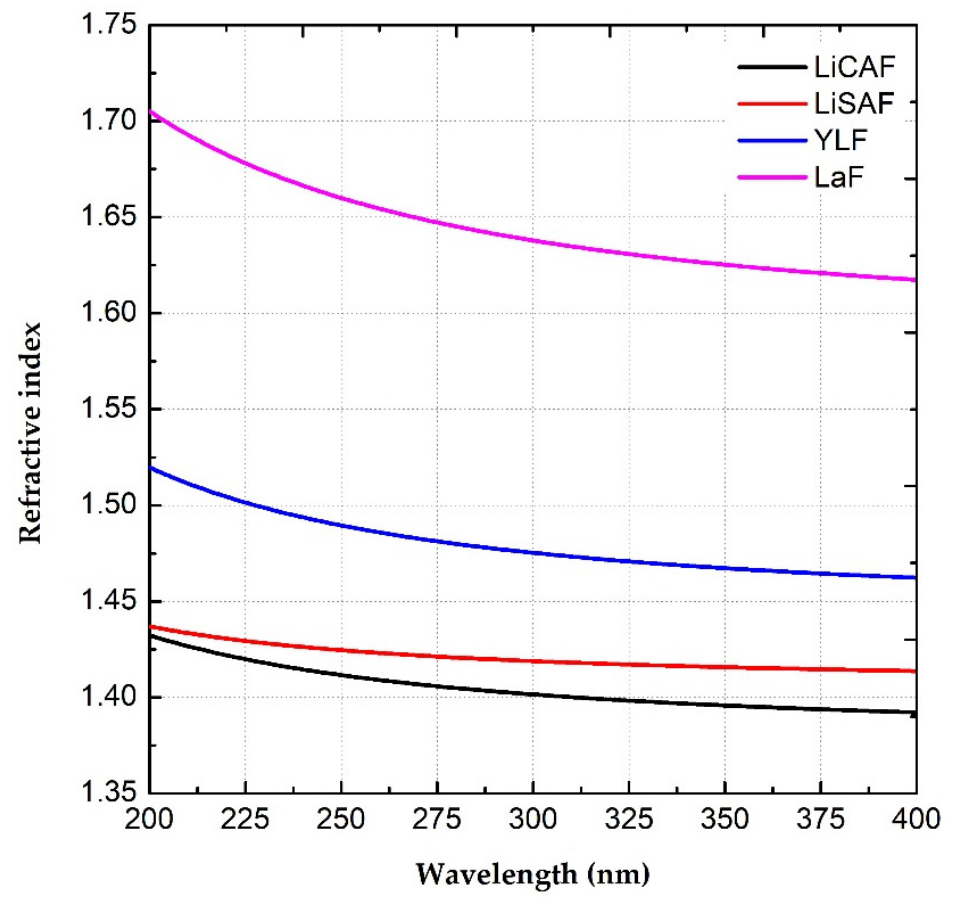

Figure 1. Linear refractive index $\left(n_{0}\right)$ of the fluoride laser materials.

Figure 2 shows the nonlinear refractive index of the fluoride host materials in the UV wavelength region. Compared to the behavior of $n_{0}$ in Figure 1 , a larger variation in $n_{2}$ is observed from LiCAF, LiSAF, and YLF with a steep decrease from $200 \mathrm{~nm}$ to about $250 \mathrm{~nm}$ before settling at a stable value from around $300 \mathrm{~nm}$. LiCAF has the smallest variation in $n_{2}$, which changed from $2 \times 10^{-16} \mathrm{~cm}^{2} / \mathrm{W}$ to $0.7 \times 10^{-16} \mathrm{~cm}^{2} / \mathrm{W}$ giving a $\Delta n_{2}$ of $1.3 \times 10^{-16} \mathrm{~cm}^{2} / \mathrm{W}$, followed by LiSAF with a $\Delta n_{2}$ of $1.7 \times 10^{-16} \mathrm{~cm}^{2} / \mathrm{W}$ and then YLF with a $\Delta n_{2}$ of $2.0 \times 10^{-16} \mathrm{~cm}^{2} / \mathrm{W}$. Interestingly, LaF exhibited a different pattern wherein its $n_{2}$ initially increases, peaking at $262 \mathrm{~nm}$, before decreasing as the wavelength becomes longer. LaF has a maximum $n_{2}$ value of $5.6 \times 10^{-16} \mathrm{~cm}^{2} / \mathrm{W}$, which occurred at $262 \mathrm{~nm}$. The larger $n_{2}$ values from LiSAF, YLF, and LaF indicate that nonlinear processes will be more evident in these materials compared to LiCAF. For the case of LaF, exciting this laser host material around $260 \mathrm{~nm}$ wavelength could lead to self-focusing effects even at lower 
excitation energies. Self-focusing effects in these laser host materials are further analyzed in the succeeding paragraphs.

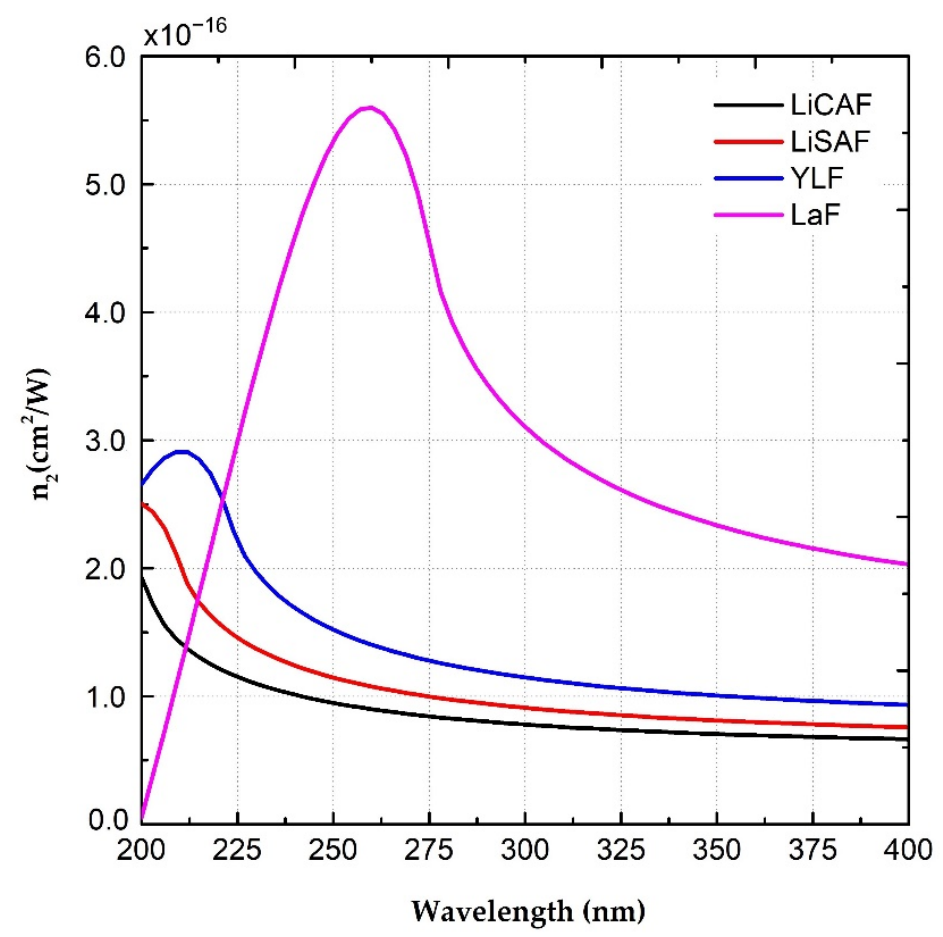

Figure 2. Nonlinear refractive index $\left(n_{2}\right)$ of the fluoride laser materials.

The spectral profile of laser emission from the $\mathrm{Ce}^{3+}$-doped fluoride materials is broad, allowing the wavelength of the laser emission to be tuned within a range of $10 \mathrm{~nm}$ to $35 \mathrm{~nm}$ [38]. Calculations were performed over this broad spectral tuning range, but detailed analysis of the nonlinear effects are considered at the peak laser emission wavelength, which is also expected to express the highest laser output energy. The peak laser emission wavelength of $\mathrm{Ce}^{3+}$-doped LiCAF, LiSAF, and LaF occurs at around $290 \mathrm{~nm}$, while it is at around $308 \mathrm{~nm}$ for Ce:YLF. Investigation of self-focusing is particularly important when scaling up the power of the laser emission towards the terawatt level, as self-focusing could easily damage the laser material at this high intensity level. The peak laser emission wavelengths and $n_{2}$ values of the fluoride laser materials at their respective peak laser emission wavelengths are summarized in Table 4.

Table 4. Peak laser emission wavelength and nonlinear refractive index $\left(n_{2}\right)$ of the fluoride laser materials at their respective peak laser emission wavelength.

\begin{tabular}{|c|c|c|}
\hline Material & $\begin{array}{l}\text { Wavelength } \\
\text { (nm) }\end{array}$ & $\begin{array}{c}\text { Nonlinear Refractive Index } \\
\times 10^{-7}\left(\mathrm{~cm}^{2} / \mathrm{W}\right)\end{array}$ \\
\hline LiCAF & 290 & 8.02 \\
\hline LiSAF & 290 & 9.41 \\
\hline $\mathrm{LaF}$ & 290 & 34.42 \\
\hline YLF & 308 & 11.1 \\
\hline
\end{tabular}

\subsection{Self-Focusing in the Fluoride Crystals}

The critical energy was determined for a UV laser beam propagating in each of the four fluoride laser host materials. The propagating laser beam is the emission from the laser material rather than the excitation beam used to achieve lasing. The laser beam is assumed to have a Gaussian shape, a pulse width of $100 \mathrm{fs}$, and a wavelength tuning range from $200 \mathrm{~nm}$ to $400 \mathrm{~nm}$. Figure 3 shows that the critical energy of each laser host material is higher when the wavelength of the laser beam is longer. As mentioned earlier, self-focusing 
will occur when the energy of the laser beam is greater than the critical energy. Table 5 summarizes the critical energy of the laser host materials at their respective peak laser emission wavelengths. Looking at the respective peak laser emission wavelengths of each of the laser host materials, LiCAF has the highest critical energy. This means that LiCAF would be able to tolerate higher laser beam energies before self-focusing sets in. The trend in critical energy for the four laser host materials is consistent with the trend observed for $n_{2}$ where LiCAF also exhibited the smallest $n_{2}$. It follows that, among the four fluoride laser host materials studied here, LiCAF has the best potential for high-power UV laser generation. On the other hand, LaF could be limited by self-focusing when higher UV laser output energies are elicited either through direct lasing or amplification.

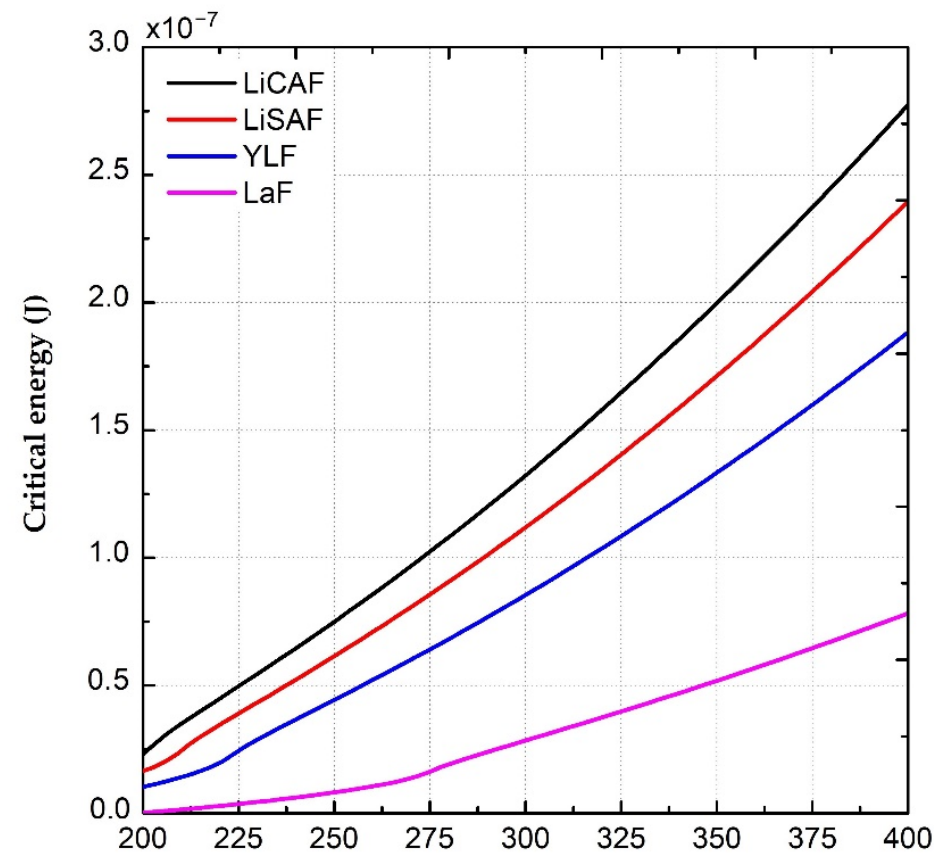

Figure 3. Critical energy, which defines the energy limit before self-focusing occurs, as a function of wavelength.

Table 5. Peak laser emission wavelength and critical energy of the fluoride laser materials at their respective peak laser emission wavelength.

\begin{tabular}{ccc}
\hline Material & $\begin{array}{c}\text { Wavelength } \\
(\mathbf{n m})\end{array}$ & $\begin{array}{c}\text { Critical Energy } \\
\times \mathbf{1 0}^{-\mathbf{7}} \mathbf{( J )}\end{array}$ \\
\hline LiCAF & 290 & 1.20 \\
LiSAF & 290 & 1.01 \\
LaF & 290 & 0.24 \\
YLF & 308 & 0.93 \\
\hline
\end{tabular}

Figure 4 shows the variation in the nonlinear refractive index $n_{2}$ for a Gaussian laser pulse with $1 \mathrm{~mJ}$ energy, $100 \mathrm{fs}$ pulse duration, and $0.5 \mathrm{~mm}$ beam radius. The calculations were performed at the peak laser emission wavelength of each of the laser materials. For a Gaussian laser beam, the intensity distribution is not uniform, and therefore $n_{2}$ also varies according to the beam's Gaussian intensity distribution. It follows that the value of $n_{2}$ is highest at the center of the beam, and it tapers off at the edge of the beam. More interestingly, the variation in $n_{2}$ is smallest for LiCAF, which means that nonlinear effects would be more homogeneous throughout the material's volume. Consequently, higher excitation energies can be used to elicit higher laser emission energies. Furthermore, laser emission would be more uniformly distributed throughout the laser material's volume as the laser threshold is achieved almost uniformly. For LaF, on the other hand, self-focusing 
could set in at areas around the peak of the Gaussian beam leading to very high intensities that could potentially damage the laser material, while the areas surrounding the peak would have lower intensities. Consequently, the energy distribution will be less uniform. To achieve a more uniform excitation and emission energy distribution, a smaller material size could be used, but this would also limit the power scalability of the LaF-based laser.

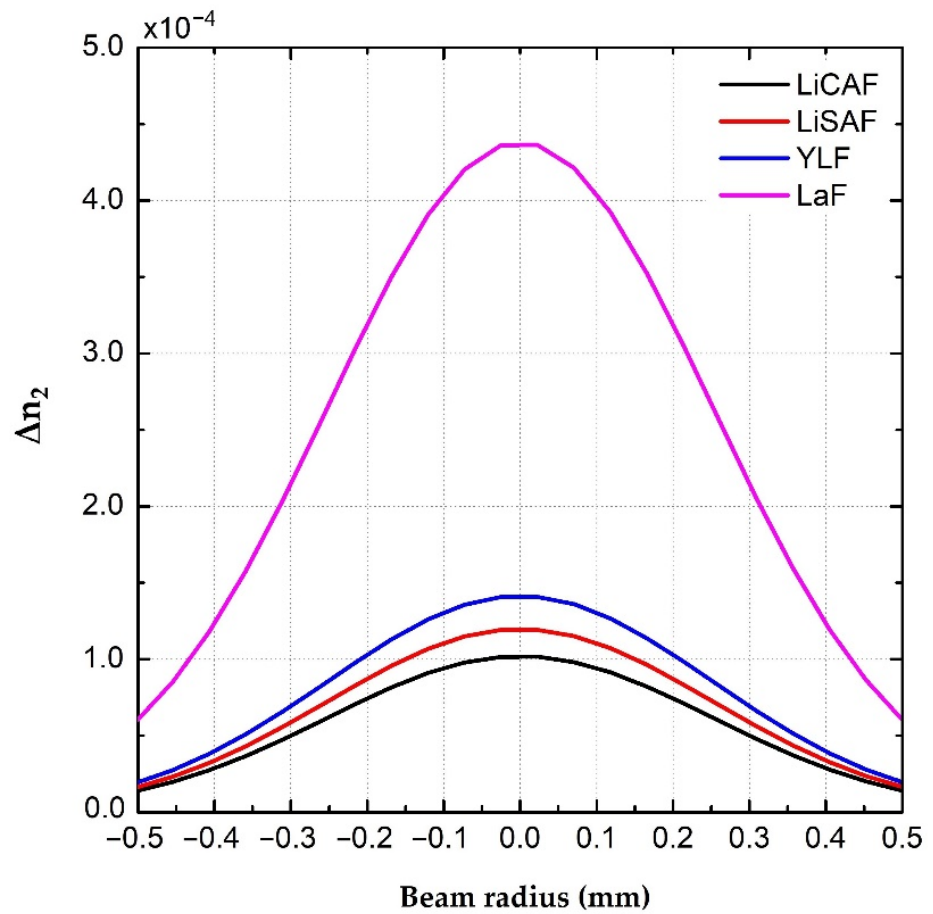

Figure 4. Variation of nonlinear refractive index $\left(\Delta n_{2}\right)$ as a function of the intensity distribution of a Gaussian laser pulse.

As $n_{2}$ leads to self-focusing, the corresponding focal length $f$ was calculated for each of the laser host materials and is shown in Figure 5. By calculating the focal length, the optimal length $d$ of the laser material is determined such that the focal point of self-focusing occurs outside the laser material. Consequently, damage to the laser material resulting from very high intensities at the focal point will be avoided. The dashed line in Figure 5 indicates the condition when the focal length of self-focusing is equal to the length of the laser material $(f=d)$. The dashed line therefore defines the upper limit of the laser material's length, meaning that a length that is longer than this limit will result in the focal point occurring inside the laser material $(f<d)$. Therefore, Figure 5 is important in deciding the suitable length of the laser material. The length of the laser material should be shorter than the focal length $(f>d)$ so that the focal point occurs outside of the laser material. The upper limit on the length of the fluoride materials studied here are $7.5 \mathrm{~mm}$ for LaF, $14 \mathrm{~mm}$ for YLF, $16 \mathrm{~mm}$ for LiSAF, and $18 \mathrm{~mm}$ for LiCAF. Notably, the LiCAF laser host material allows for a longer length to be used. This longer length is advantageous for the development of high-power, ultrashort lasers using, for example, multipass laser oscillator and amplifier configurations that result in higher laser emission energies.

In order to achieve enough laser fluence to satisfy the laser threshold and enable lasing, the excitation beam radius is usually reduced. As the beam radius is reduced, the intensity increases, triggering self-focusing. Therefore, the focal length was calculated as a function of beam radius from $0.1 \mathrm{~mm}$ to $0.6 \mathrm{~mm}$. The calculations assumed a Gaussian beam with $1 \mathrm{~mJ}$ energy and $100 \mathrm{fs}$ pulse duration. Figure 6 shows the calculation results. Once again, the dashed line indicates the limit in the laser material's length that defines whether the focal point occurs inside or outside the material. The dashed line also defines the lower limit on the size of the excitation beam. In order for the focal point to occur outside the laser material, the radius of the excitation beam should be larger than the limit (dashed 
line). The lower limit on the excitation beam radius is $0.55 \mathrm{~mm}$ for LaF, $0.42 \mathrm{~mm}$ for YLF, $0.39 \mathrm{~mm}$ for LiSAF, and $0.37 \mathrm{~mm}$ for LiCAF. These results show that a smaller beam radius can be used for LiCAF, effectively achieving a higher laser fluence.

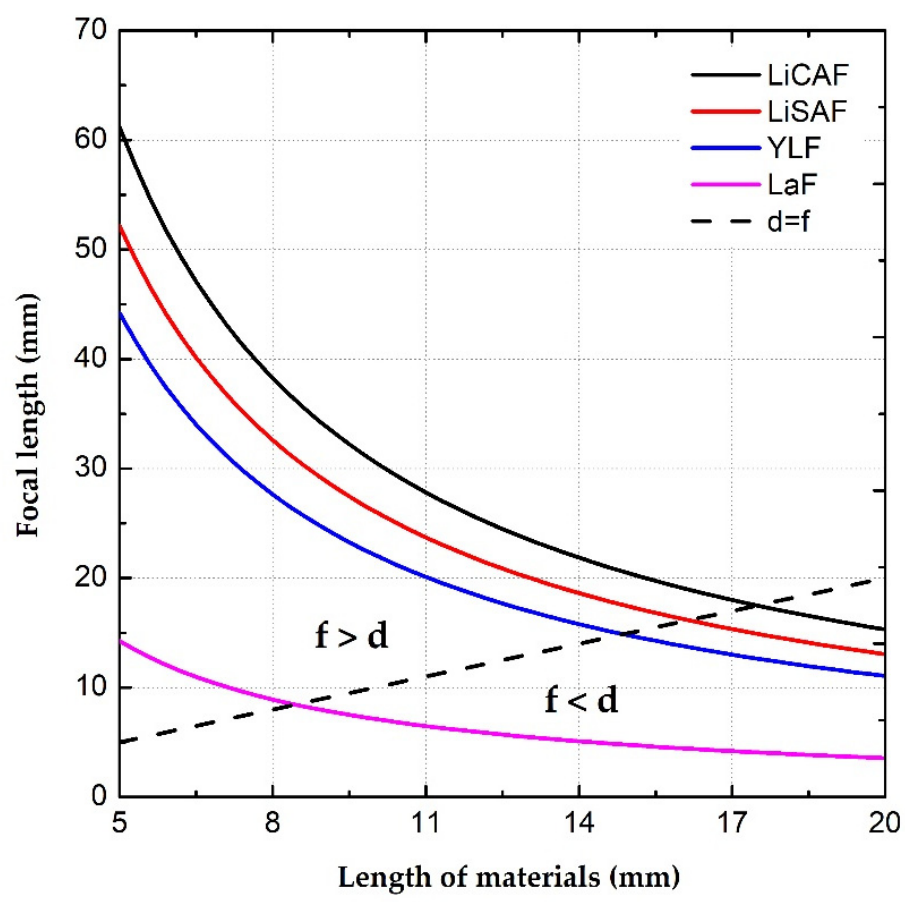

Figure 5. Focal length as a function of the length of the laser host material. The dashed line indicates the condition when the focal length of self-focusing is equal to the length of the laser material $(f=d)$.

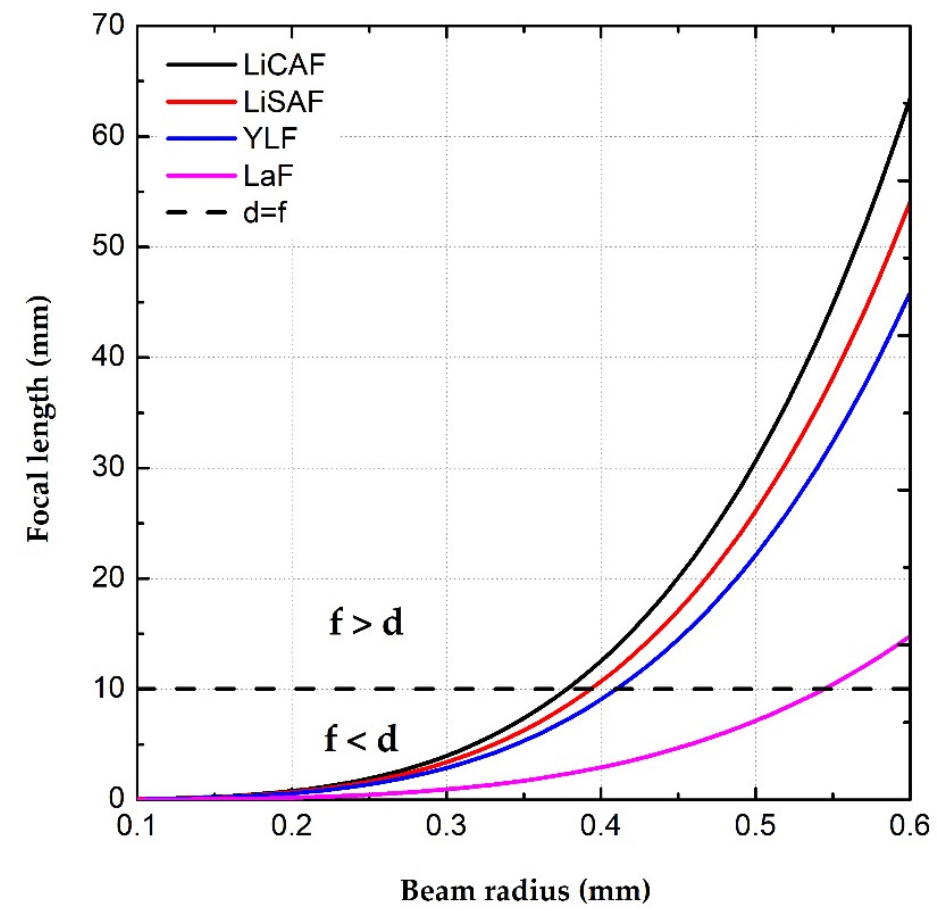

Figure 6. Focal length as a function of the beam radius. The dashed line indicates the limit in the laser material's length that defines whether the focal point occurs inside or outside the material. It also defines the lower limit on the size of the excitation beam. 


\section{Conclusions}

In conclusion, the nonlinear refractive index $\left(n_{2}\right)$ of different fluoride laser materials including LiCAF, LiSAF, YLF, and LaF were determined for the first time using the KramersKrönig relation model in the UV region. Among the laser host materials reported to lase in the UV region when doped with $\mathrm{Ce}^{3+}$ ions, LiCAF exhibited the smallest $n_{2}$ in the UV wavelength range from $200 \mathrm{~nm}$ to $400 \mathrm{~nm}$. Consequently, a higher laser intensity can be achieved before self-focusing within the laser material and its associated damaging effects sets in. In addition, a longer material length can be used while still ensuring that the focal point of the self-focused laser beam occurs outside of the laser material. A longer material length is advantageous for the development of high-power, ultrashort lasers using, for example, multipass laser oscillator and amplifier configurations that result in higher laser emission energies. The small $n_{2}$ also means that a smaller beam radius can be used, thereby effectively achieving a higher laser fluence. The variation in $n_{2}$ of LiCAF is also relatively flat across the varying intensity of a Gaussian laser beam. These results indicate that LiCAF is more advantageous as a laser host material in the UV region. In general, results of this work could be used as a guide when determining the optimal parameters of the laser material for the development of an ultrashort pulse and ultrahigh-power laser.

Author Contributions: Conceptualization, M.H.P. and M.C.-R.; Formal analysis, D.V.N., M.H.P. and M.C.-R.; Funding acquisition, M.H.P. and M.C.-R.; Investigation, D.V.P., D.V.N., T.X.N. and K.A.T.D.; Methodology, D.V.N. and M.H.P.; Project administration, M.H.P.; Resources, M.H.P.; Visualization, D.V.P., D.V.N., T.X.N., K.A.T.D. and Q.M.L.; Writing-original draft, D.V.N., M.H.P. and M.C.-R.; Writing-review and editing, D.V.P., T.X.N., K.A.T.D. and Q.M.L. All authors have read and agreed to the published version of the manuscript.

Funding: This research was funded by Vietnam National Foundation for Science and Technology Development (NAFOSTED 103.03-2019.365).

Institutional Review Board Statement: Not applicable.

Informed Consent Statement: Not applicable.

Data Availability Statement: The data presented in this study are available from the corresponding authors upon reasonable request.

Conflicts of Interest: The authors declare no conflict of interest. The funders had no role in the design of the study; in the collection, analyses, or interpretation of data; in the writing of the manuscript, or in the decision to publish the results.

\section{References}

1. Malinauskas, M.; Zukauskas, A.; Hasegawa, S.; Hayasaki, Y.; Mizeikis, V.; Buividas, R.; Juodkazis, S. Ultrafast laser processing of materials: From science to industry. Light Sci. Appl. 2016, 5, 3-5. [CrossRef] [PubMed]

2. Xiong, Z.; Li, H.; Kunwar, P.; Zhu, Y.; Ramos, R.; Mcloughlin, S.; Winston, T.; Ma, Z.; Soman, P. Femtosecond laser induced densification within cell-laden hydrogels results in cellular alignment. Biofabrication 2019, 11, 035005. [CrossRef] [PubMed]

3. Jeppson, S.; Kukreja, R. Capturing ultrafast magnetization phenomenon using femtosecond X rays. APL Mater. 2021, 9, 100702. [CrossRef]

4. Zhang, B.; Wang, L.; Chen, F. Recent advances in femtosecond laser processing of $\mathrm{LiNbO}_{3}$ crystals for photonic applications. Laser Photonics Rev. 2020, 14, 1900407. [CrossRef]

5. Wang, X.; Yu, H.; Li, P.; Zhang, Y.; Wen, Y.; Qiu, Y.; Liu, Z.; Li, Y.P.; Liu, L. Femtosecond laser-based processing methods and their applications in optical device manufacturing: A review. Opt. Laser Technol. 2021, 135, 106687. [CrossRef]

6. Xiao, Y.; Zhang, X.; Li, R.; Liu, H.; Zhou, N.; Zhang, J. Single-crystal $\mathrm{LaB}_{6}$ field emission array is rapidly fabricated by ultraviolet femtosecond laser and its field electronic structure characteristics. Vacuum 2021, 184, 109987. [CrossRef]

7. Lai, Y.; Xue, Y.; Côté, C.; Liu, X.; Laramée, A.; Jaouen, N.; Légaré, F.; Tian, L.; Liang, J. Single-shot ultraviolet compressed ultrafast photography. Laser Photonics Rev. 2020, 14, 2000122. [CrossRef]

8. Almeida, G.F.B.; Martins, R.J.; Siqueira, J.P.; Almeida, J.M.P.; Rodrigues, J.J., Jr.; Mendonça, C.R. Nonlinear optical waveguides inscribed by fs-laser in organic crystal for broadband second harmonic generation of UV pulses. Opt. Mater. 2018, 83, 229-232. [CrossRef]

9. Baryshnikov, V.I.; Sarukura, N.; Segawa, Y. Solid state tunable lasers with nanosecond flashlamp pumping. In Proceedings of the Laser Optics' 95: Solid State Lasers, St. Petersburg, Russia, 27 June-1 July 1996; Volume 2772, pp. 74-77. 
10. Ehrlich, D.J.; Moulton, P.F.; Osgood, R.M. Optically pumped Ce:LaF 3 laser at 286 nm. Opt. Lett. 1980, 5, 339-341. [CrossRef]

11. Rambaldi, P.; Moncorgé, R.; Wolf, J.P.; Pédrini, C.; Gesland, J.Y. Efficient and stable pulsed laser operation of Ce:LiLuF 4 around 308 nm. Opt. Commun. 1998, 146, 163-166. [CrossRef]

12. Minh, P.H.; Cadatal-Raduban, M.; Duong, P.V.; Tu, N.X.; Mui, L.V.; Yamanoi, K.; Shimizu, T.; Sarukura, N.; Hung, N.D. Tunable narrow linewidth picosecond pulses from a single grating gain-switched Ce:LiCAF laser. Laser Phys. 2018, $28,085802$.

13. McGonigle, A.J.S.; Coutts, D.W.; Girard, S.; Moncorgé, R. A 10 kHz Ce:LiSAF laser pumped by the sum-frequency-mixed output of a copper vapour laser. Opt. Commun. 2001, 193, 233-236. [CrossRef]

14. Diep, N.V.; Cadatal-Raduban, M.; Duong, P.V.; Tu, N.X.; Thu, V.V.; Minh, P.H. Tunable dual wavelength and narrow linewidth laser using a single solid-state gain medium in a double Littman resonator. Opt. Commun. 2021, 496, 127131.

15. Ono, S.; Suzuki, Y.; Kozeki, T.; Murakami, H.; Ohtake, H.; Sarukura, N.; Sato, H.; Machida, S.; Shimamura, K.; Fukuda, T. High-energy, all-solid-state, ultraviolet laser power-amplifier module design and its output-energy scaling principle. Appl. Opt. 2002, 41, 7556-7560. [CrossRef]

16. Liu, Z.; Kozeki, T.; Suzuki, Y.; Sarukura, N.; Shimamura, K.; Fukuda, T.; Hirano, M.; Hosono, H. Ultraviolet femtosecond pulse amplification with high gain using solidstate, broad-band gain medium $\mathrm{Ce}^{3+}: \mathrm{LiCaAlF}_{6}$. Jpn. J. Appl. Phys. 2001, 40, 2308-2309. [CrossRef]

17. Gabayno, J.L.; Cadatal-Raduban, M.; Pham, M.; Yamanoi, K.; Estacio, E.; Garcia, W.; Nakazato, T.; Shimizu, T.; Sarukura, N.; Suyama, T.; et al. Amplification of ultraviolet femtosecond pulse by a micro-pulling down method-grown Ce:LiCAF crystal in a prismatic cell-type, side-pumping configuration. Jpn. J. Appl. Phys. 2009, 48, 120213. [CrossRef]

18. Liu, Z.; Kozeki, T.; Suzuki, Y.; Sarukura, N.; Shimamura, K.; Fukuda, T.; Hirano, M.; Hosono, H. Chirped-pulse amplification of ultraviolet femtosecond pulses by use of $\mathrm{Ce}^{3+}: \mathrm{LiCaAlF}_{6}$ as a broadband, solid-state gain medium. Opt. Lett. 2001, 26, 301-303. [CrossRef] [PubMed]

19. Sharp, A.; Kitzler, O.; Fuerbach, A.; Spence, D.J.; Coutts, D.W. Femtosecond ultraviolet pulses generated directly from a mode-locked Ce:LiCAF lasser. In Proceedings of the Optical Society of America, Sydney, NSW, Australia, 2-6 August 2020. C7A_2.

20. Cadatal-Raduban, M.; Minh, P.H.; Duong, P.V.; Duong, B.T.T.; Yamanoi, K.; Takeda, K.; Melvin, J.F.E.; Mui, L.V.; Shimizu, T.; Hung, N.D.; et al. Total internal reflection-based side-pumping configuration for terawatt ultraviolet amplifier and laser oscillator development. Appl. Phys. B 2018, 124, 125. [CrossRef]

21. Seidel, M.; Brons, J.; Arisholm, G.; Fritsch, K.; Pervak, V.; Pronin, O. Efficient High-Power Ultrashort Pulse Compression in Self-Defocusing Bulk Media. Sci. Rep. 2017, 7, 1410. [CrossRef]

22. Akbari, R.; Major, A. Kerr-lens mode locking of a diode-pumped Yb:KGW laser using an additional intracavity Kerr medium. Laser Phys. Lett. 2018, 15, 085001. [CrossRef]

23. Beygi, M.G.; Karimzadeh, R.; Dashtdar, M. Nonlinear refractive index measurement by Fresnel diffraction from phase object. Opt. Laser Technol. 2015, 66, 151-155. [CrossRef]

24. Sheik-Bahae, M.; Hutchings, D.C.; Hagan, D.J.; Van Stryland, E.W. Dispersion of bound electronic nonlinear refraction. IEEE J. Quantum Electron. 1991, 27, 1296. [CrossRef]

25. Major, A.; Yoshino, F.; Nikolakakos, I.; Aitchison, J.S.; Smith, P.W.E. Dispersion of the nonlinear refractive index in sapphire. Opt. Lett. 2004, 29, 602-604. [CrossRef]

26. Cadatal-Raduban, M.; Minh, P.H.; Mui, L.V.; Hung, N.D.; Sarukura, N. Ultrashort pulse generation in Ce:LiCAF ultraviolet laser. In Numerical Simulations in Engineering and Science; IntechOpen: London, UK, 2018; pp. 135-162.

27. Mui, L.V.; Melvin, J.F.E.; Gabayno, J.L.F.; Minami, Y.; Yamanoi, K.; Shimizu, T.; Sarukura, N.; Minh, P.H.; Hung, N.D.; Steenbergen, K.G.; et al. Direct band gap tunability of the LiYF4 crystal through high-pressure applications. Comput. Mater. Sci. 2018, 153, $431-437$.

28. Nafulawabululu, E.; Nyawere, P.W.O.; Barasa, D.B. First-principles calculations of structural, electronic and elastic properties of tysonite lanthanum fluoride $\left(\mathrm{LaF}_{3}\right)$. J. Res. Environ. Earth Sci. 2018, 4, 36-40.

29. Sheik-Bahae, M.; Stryland, E.W.V. Optical nonlinearities in the transparency region of bulk semiconductors. In Semiconductors and Semimetals; Academic Press: San Diego, CA, USA, 1999; Volume 58, pp. 257-318.

30. Fibich, G.; Gaeta, A.L. Critical power for self-focusing in bulk media and in hollow waveguides. Opt. Lett. 2000, 25, 335-337. [CrossRef]

31. Kriso, C.; Kress, S.; Munshi, T.; Grossmann, M.; Bek, R.; Jetter, M.; Michler, P.; Stolz, W.; Koch, M.; Rahimi-Iman, A. Microcavityenhanced Kerr nonlinearity in a vertical-external-cavity surface-emitting laser. Opt. Express 2019, 27, 11914-11929. [CrossRef] [PubMed]

32. Shimamura, K.; Sato, H.; Bensalah, A.; Machida, H.; Sarukura, N.; Fukuda, T. Growth of LiCaAlF single crystals with an extended diameter and their optical characterizations. J. Alloy. Compd. 2002, 343, 204-210. [CrossRef]

33. Weber, M.J. CRC Handbook of Laser Science and Technology Supplement 2 Optical Materials; CRC Press: Boca Raton, FL, USA, $1994 ;$ p. 599.

34. Barnes, N.P.; Gettemy, D.J. Temperature variation of the refractive indices of yttrium lithium fluoride. J. Opt. Soc. Am. 1980, 70, 1244-1247. [CrossRef]

35. Laiho, R.; Lakkisto, M. Investigation of the refractive indices of $\mathrm{LaF}_{3}, \mathrm{CeF}_{3}, \mathrm{PrF}_{3}$ and $\mathrm{NdF}_{3}$. Philos. Mag. B 1983, 48, 203-207. [CrossRef]

36. Wellmann, B.; Spence, D.J.; Coutts, D.W. Dynamics of solid-state lasers pumped by mode-locked lasers. Opt. Express 2015, 23, 4441. [CrossRef] [PubMed] 
37. Woods, B.W.; Payne, S.A.; Marion, J.E.; Hughes, R.S.; Davis, L.E. Thermomechanical and thermo-optical properties of the $\mathrm{LiCaAlF}_{6}: \mathrm{Cr}^{3+}$ laser material. J. Opt. Soc. Am. B 1991, 8, 970-977. [CrossRef]

38. Coutts, D.W.; McGonigle, A.J.S. Cerium-Doped Fluoride Lasers. IEEE J. Quantum Electron. 2004, 40, 1430-1440. [CrossRef] 